\title{
AlineAMiEnTo Y APLICACIONES DEL NEUTRÓGRAFO DEL REACTOR RP-10
}

\section{Alignment and applications of the NEUTROGRAPH OF THE RP-10 REACTOR}

\author{
Alcides López y Javier Gago \\ Dirección de Investigación y Desarrollo, Instituto Peruano de Energía Nuclear, Av. Canadá \\ i470, Lima 4I, Perú
}

\begin{abstract}
RESUMEN
Se presenta el proceso de alineamiento del eje óptico del sistema neutrográfico con el eje óptico del haz de neutrones del ducto número 4 del reactor nuclear RP-10, asistido por la técnica radiográfica que facilita la ubicación del eje óptico del haz de neutrones en el espacio mediante registro fotográfico y permite posteriormente el alineamiento del neutrógrafo. En las aplicaciones, se aprovecha el elevado coeficiente de absorción neutrónica que tienen los elementos químicos ligeros y el alto contraste de materiales con densidades similares. El neutrógrafo tiene amplia variedad de aplicaciones, algunas de ellas son: el análisis de combustibles nucleares, la detección de materiales hidrogenados y orgánicos, la detección de fallas en álabes de turbinas, la corrosión en componentes de aeronaves, el control de calidad de materiales cerámicos, la detección de drogas y materiales explosivos (útiles en la industria pirotécnica y de municiones), el estudio de materiales arqueológicos, la detección de películas lubricantes en sistemas de rodamiento, así como procesos dinámicos de lubricación y combustión, entre otros. En el presente trabajo se muestran variados ejemplos aplicativos obtenidos con el reactor RP-10.
\end{abstract}

Descriptores: neutrografia, reactor nuclear, ensayo no destructivo

\begin{abstract}
We present here the process of optical axis alignment of the neutrographic system with the optical axis of the neutron beam from window 4 of the nuclear reactor RP-10. This process is done with the aid of a radiographic technique, which facilitates locating the optical axis of the neutron beam in space by a photographic record, thus allowing for later alignment of the neutrograph. The high neutronic absorption coefficient of light chemical elements and the high contrast of materials with similar density are an advantage for applications. The neutrograph has a wide variety of applications, among them: the analysis of nuclear fuels, the detection of hydrogenated and organic materials, the detection of flaws in turbine blades, the detection of corrosion in aircraft components, quality control of ceramic materials, detection of drugs and explosive materials (used in the pyrotechnic and ammunition industry), the study of archaeological materials, the detection of lubricating films in bearing systems, as well as dynamic processes of lubrication and combustion, among others. In this work we present a variety of examples of applications with the reactor RP-10.
\end{abstract}

Keywords: neutrography, nuclear reactor, non-destructive essay 


\section{INTRODUCCIÓN}

El neutrógrafo, como cualquier otro sistema óptico, requiere una instalación apropiada para que tenga un funcionamiento eficiente. Dicha instalación consiste básicamente en el alineamiento óptico, cuyo procedimiento consta de las siguientes fases: 1 ) ubicación de la fuente, 2) orientación de la fuente, 3) análisis de la distribución espacial de la radiación, 4) medición de la potencia y 5) coincidencia del eje óptico del haz de radiación con el eje óptico del instrumento [1]. Dada las características de la radiación neutrónica producida por el reactor nuclear RP-10 del Instituto Peruano de Energía Nuclear (IPEN), la caracterización ha requerido de técnicas radiográficas para la localización espacial del haz neutrónico, seguida de la medición del flujo neutrónico y la aplicación de los sistemas de protección y seguridad radiológica. En la parte de las aplicaciones, se tiene en cuenta que la neutrografía o radiografía neutrónica es una técnica de ensayo no destructivo que permite ver o fotografiar el interior de estructuras sólidas por medio de un haz de neutrones, y juega un importante rol en la medicina, biología y ciencia de los materiales, aportando importantes aplicaciones en forma de imágenes. La característica principal de esta técnica es la elevada penetración a través de materiales industriales basados en acero, aluminio, zirconio, etc. Los neutrones son eficientemente absorbidos solo por unos pocos elementos ligeros como el hidrógeno, boro, cadmio, samario y gadolinio [2-4]. Por ejemplo, los materiales orgánicos o el agua atenúan fuertemente a los neutrones debido a su alto contenido de hidrógeno, mientras que muchas estructuras hechas con aluminio o acero resultan casi transparentes, y además muestran un alto contraste de materiales con similares densidades [2-6].

La neutrografía es una técnica similar a la radiografía por rayos $\mathrm{X}$ o gamma y tiene algunas ventajas particulares en el campo nuclear, aeroespacial y en la industria de plásticos y látex. También ha sido adoptada como un método estándar para el estudio comparativo de absorción de agua en madera, polímeros y compuestos de madera-polímero, donde se miden las variaciones de la densidad óptica de dichos materiales $[7,8]$, entre otros.
Entre las aplicaciones típicas de la radiografía neutrónica, podemos mencionar al análisis de combustibles nucleares, la detección de materiales hidrogenados y orgánicos, la detección de fallas en partes de máquinas, la corrosión en componentes de aeronaves, el controldecalidaddematerialescerámicos, la detección de drogas y materiales explosivos (útiles en la industria pirotécnica y de municiones), el estudio de materiales arqueológicos, la detección de películas lubricantes en sistemas de rodamiento, así como procesos dinámicos de lubricación y combustión, entre otros. La neutrografía, al permitir alcanzar una importante resolución de imagen $(50 \mu \mathrm{m})$, ha hecho posible el desarrollo de la neutrografía microscópica, que requiere el uso de microscopios de luz para la amplificación correspondiente de la imagen, y ha obtenido resultados importantes en el estudio de cortes histológicos $[9,10]$.

Los neutrones, al interactuar de manera diferente con la materia, ofrecen información complementaria a los observados con rayos $\mathrm{X}$ o gamma. Al requerir de una fuente de neutrones, las aplicaciones se ven bastante limitadas y solo algunas instituciones, como el IPEN, tienen la capacidad de ofrecer esta facilidad neutrográfica al usuario.

\section{MÉTODO EXPERIMENTAL}

El reactor nuclear RP-10 del IPEN consta, en su primera parte, de un núcleo sumergido en una piscina que contiene agua como moderador. El conducto de irradiación número 4 consta de un colimador cónico, con sus paredes recubiertas de cadmio (fig.1). Una vista en perspectiva del neutrógrafo puede ser apreciada en la figura 2, donde se muestra en primer plano los muros de protección. El colimador va dentro de la piscina y puede ser desplazado en forma automática desde su posición normal, que está a $50 \mathrm{~cm}$, hasta la posición de irradiación, movimiento que se hace por medios mecánicos (fig. 3).

La segunda parte del colimador consta de dos cilindros recubiertos de cadmio, cuyo objetivo es absorber los neutrones térmicos no colimados que salen de la base del colimador. Al final de dichos cilindros se encuentra un obturador de plomo y cadmio C (fig. 3), que cubre la apertura del conducto y es controlado por un sistema neumático. 
El blindaje al exterior del conducto está conformado por paredes de hormigón, formando un recinto cerrado en cuyo interior se encuentra un atenuador de neutrones A. Entre este atenuador y el núcleo $\mathrm{N}$ se encuentran la placa fotográfica y su placa escintiladora P, el objeto a ser neutrografiado M y la compuerta C. La potencia nominal de operación del RP-10 fue de $10 \mathrm{MW}$, con un flujo aproximado de $6 \times 10^{6} \mathrm{n} / \mathrm{cm}^{2} \mathrm{~s}$; el diámetro del haz neutrónico fue de $25 \mathrm{~cm}$.

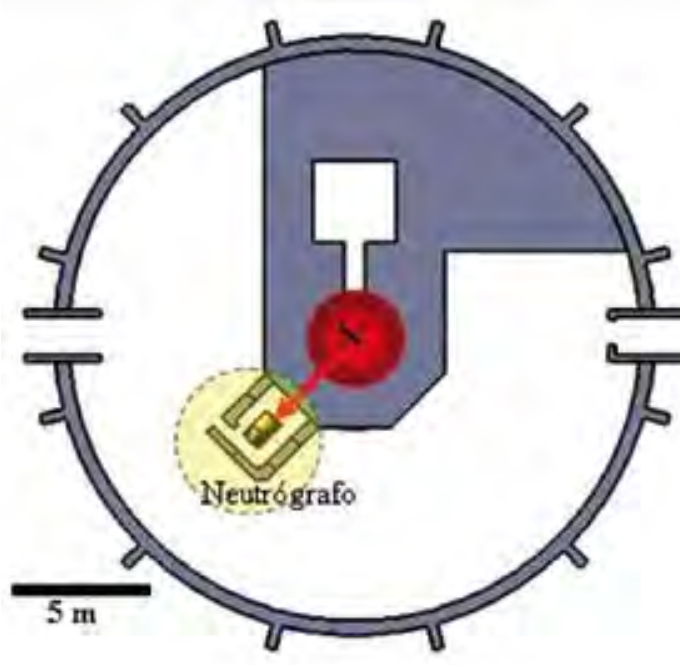

Figura 1: Vista superior en corte del reactor nuclear RP-10 e instalaciones de neutrografía.

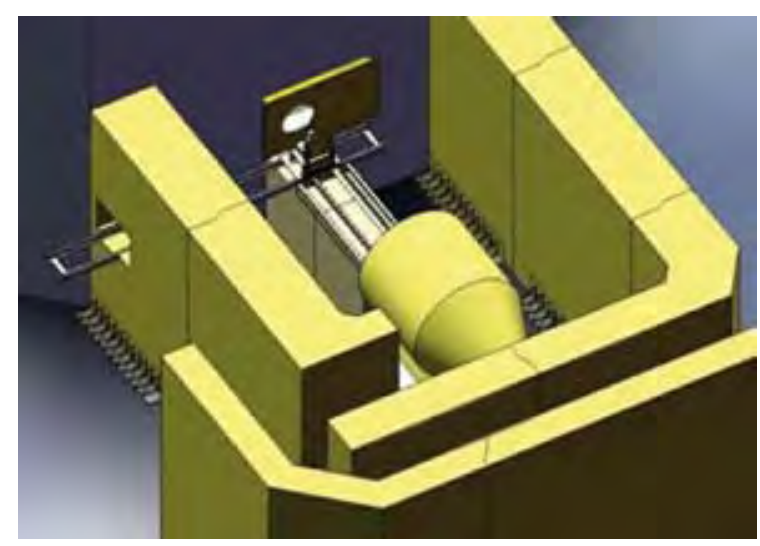

Figura 2: Vista panorámica del neutrógrafo asociado al reactor nuclear $R P-10$, rodeado de sus paredes de protección radiológica.

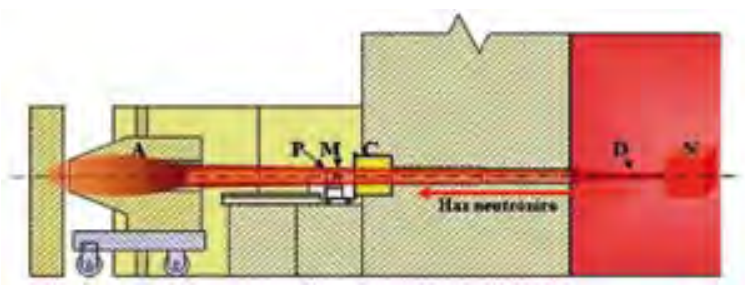

Figura 3: Vista esquemática del sistema óptico del neutrógrafo, mostrando el eje óptico y los componentes ópticos principales.

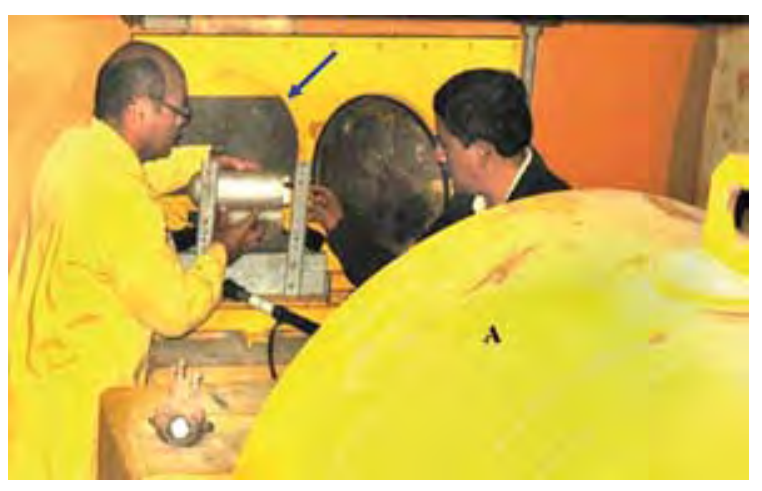

Figura 4: Instalación de una cámara de ionización en la salida del haz de neutrones del RP-10 para la correspondiente prueba neutrográfica. Delante, se aprecia el atenuador de neutrones $A$.

\section{RESULTADOS Y DISCUSIÓN}

Para la caracterización del haz de neutrones de la salida número 4 del reactor RP-10 (fig. 4), en donde se puede observar la compuerta de salida de los neutrones (flecha azul), se tuvo por conveniente hacer dos neutrografías fuera del centro, con el fin de estimar la distribución radial del flujo de neutrones a una distancia de $40 \mathrm{~cm}$ de la salida del ducto, de manera que también estuviera incluida la zona de penumbra o dispersión. Para dicha caracterización, se hizo el centrado de los arcos de circunferencia usando la opción de centrado del software "Diffraction". La figura 5a corresponde al registro neutrográfico de un sector del haz de neutrones con un flujo aproximado de $6 \times 10^{6} \mathrm{n} / \mathrm{cm}^{2}$ s. Se aprecia el centro del haz $\mathrm{C}$ y las flechas radiales. La figura $5 \mathrm{~b}$ muestra la distribución del flujo neutrónico de un sector del haz y la figura $5 c$ presenta el perfil 3D de la distribución del flujo neutrónico de un sector del mismo sector del haz. El color rojo representa el flujo más elevado, mientras que el azul y violeta los flujos más bajos, correspondientes a la zona de penumbra.

Para la medición del perfil de intensidad radial promedio, se aplicó el software ImageJ. En la figura 6 se muestra el resultado de promediar cinco perfiles obtenidos en ambas neutrografías. Allí se pueden apreciar tres zonas: la Zona I, que corresponde a un haz de $7,5 \mathrm{~cm}$ de radio de flujo neutrónico muy regular; la Zona II, zona de dispersión, que envuelve a la Zona I, en la que decae el flujo neutrónico suavemente con una tendencia lineal de hasta un $70 \%$ y alcanza un radio de $14,5 \mathrm{~cm}$; y la Zona III, 

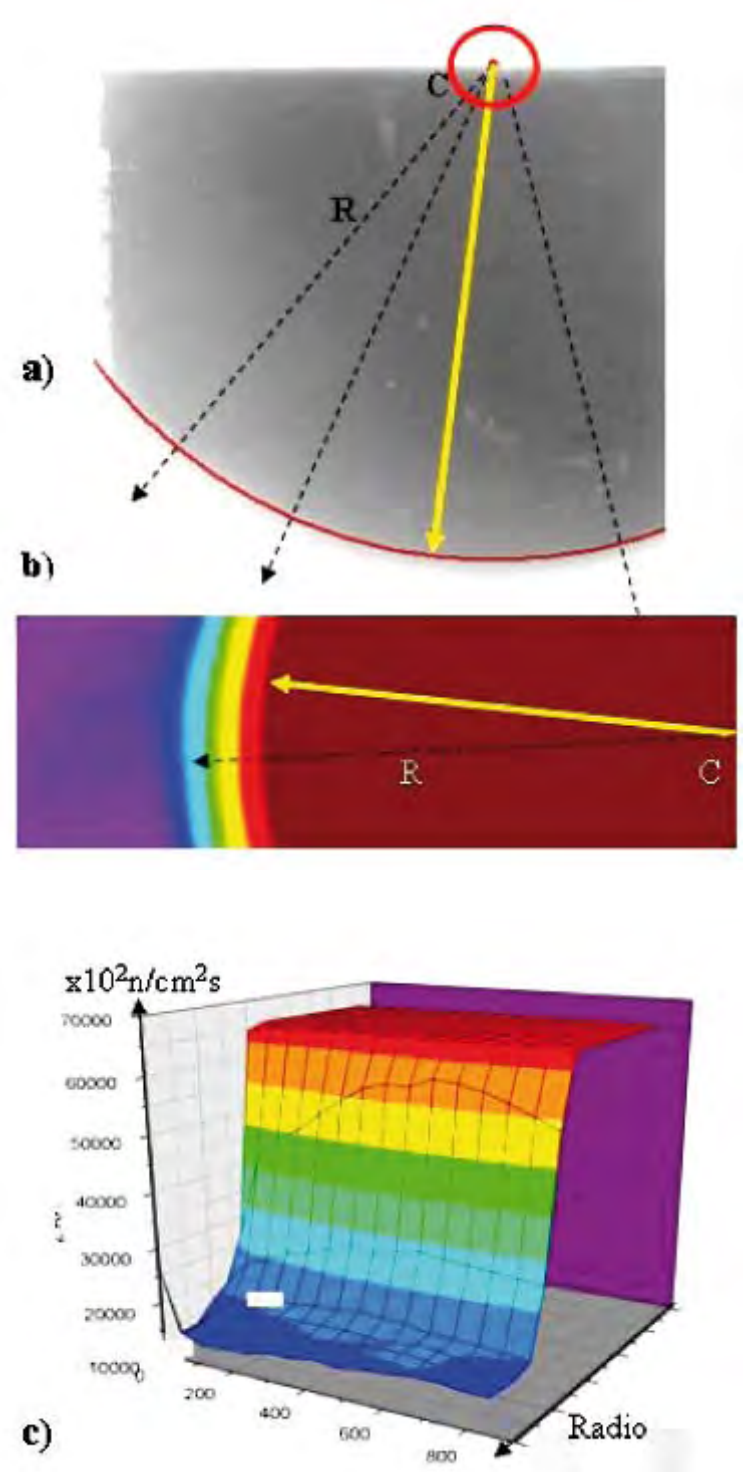

Figura 5: a) Neutrografía del haz, b) mapeo del flujo neutrónico de un sector del haz y c) perfil 3D de flujo neutrónico de un sector del haz.

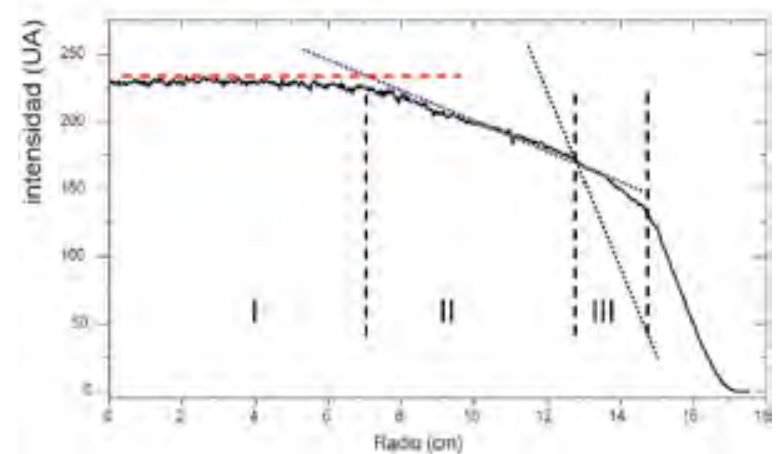

Figura 6: Perfil promedio de intensidades del haz de neutrones a una distancia de $40 \mathrm{~cm}$ de la salida del ducto nro. 4 del RP-10, operando a $10 \mathrm{MW}$. que corresponde a la penumbra o dispersión alta, y abarca desde un radio de $14,5 \mathrm{~cm}$ hasta decaer casi completamente con una tendencia lineal a un radio de $17 \mathrm{~cm}$. La tabla 1 resume las características del haz neutrónico.

Tabla 1: Distribución del flujo neutrónico en el haz número 4 del Reactor RP-10 operando a $10 \mathrm{MW}$.

\begin{tabular}{|c|c|c|c|}
\hline Zona & $\begin{array}{l}\text { Radio de } \\
\text { zona } \\
(\mathrm{cm})\end{array}$ & $\begin{array}{c}\text { Flujo } \\
\text { neutrónico } \\
10^{6} \mathrm{n} / \mathrm{cm}^{2} \mathrm{~s}\end{array}$ & Tendencia \\
\hline I & 0 a 7,5 & 6 & Constante \\
\hline II & 7,5 a 14,5 & 6 a 4,2 & Lineal \\
\hline III & 14,5 a 17 & 4,2 a 0 & Lineal \\
\hline
\end{tabular}

A fin de evitar efectos de la radiación gamma, que frecuentemente acompaña a la radiación neutrónica e interfiere con las neutrografías, se ha procedido a evaluar el grado de adición sobre la de los neutrones. En la figura 7, se aprecian dos zonas de una neutrografía: la más oscura corresponde a la zona sin filtro de radiación gamma, la más clara ha sido filtrada mediante una placa de plomo de $12 \mathrm{~mm}$ de espesor. La razón de absorción de estos rayos ha resultado ser del $22 \%$, debiendo considerarse que el restante $78 \%$ corresponde a la radiación neutrónica, principalmente.
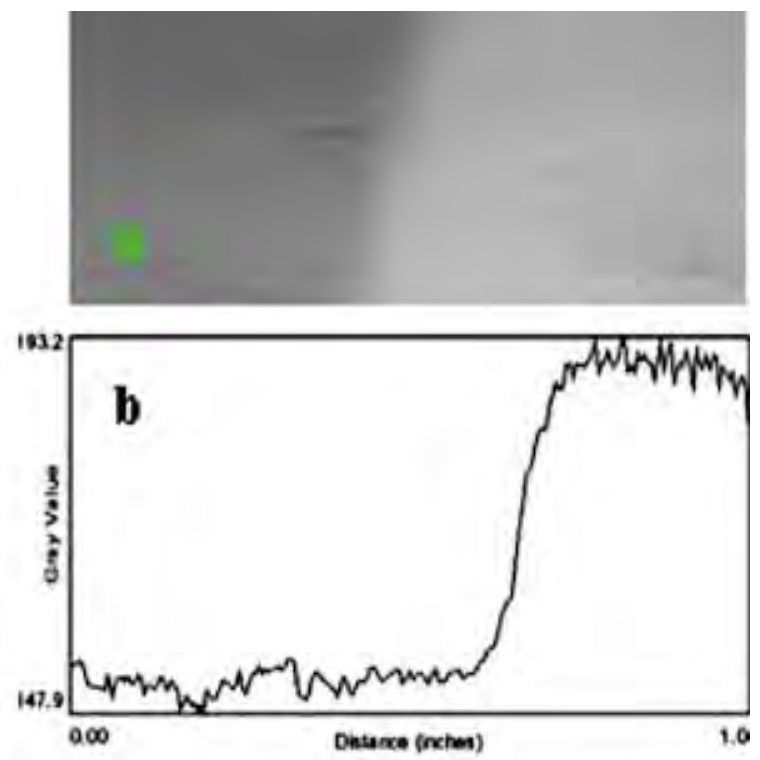

Figura 7: a) Registro fotográfico del haz de neutrones sin y con filtro de rayos gamma y b) Espectro de absorbancia de neutrones y rayos gamma. 


\section{APLICACIONES}

Entre las aplicaciones prácticas del sistema neutrográfico, se muestran algunas donde sobresalen las ventajas de esta técnica. Por ejemplo, los materiales hidrogenados ofrecen alto contraste, como puede observarse en la figura 8 , que muestra a una cucaracha neutrografiada. El alto contraste producido en la cabeza y el abdomen nos informa la abundancia de agua, mientras que en la parte inicial del abdomen un bajo nivel de agua, y los músculos del tórax y extremidades ligeramente hidrogenados.

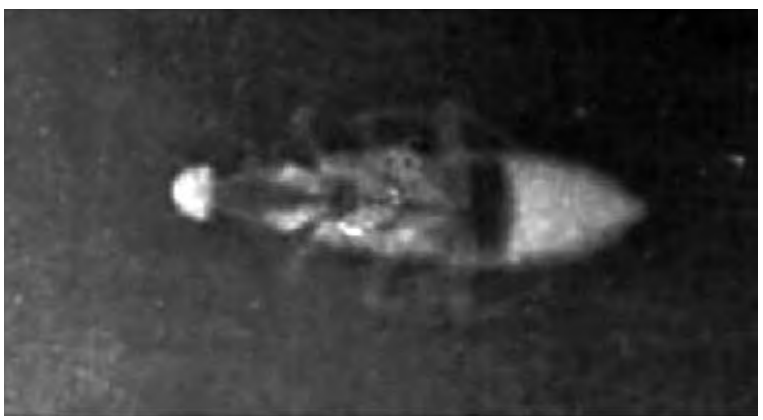

Figura 8: Neutrografía en tamaño natural de una cucaracha mostrando zonas altamente hidrogenadas en color claro.

En los cuerpos biológicos, se aprecia marcadamente las regiones con abundancia de agua, por lo que la técnica se acerca a una hidroscopia, que aplicada al estudio de plantas puede servir para identificar la localización interna de zona húmedas o con abundante líquido. En la figura 9, se aprecian neutrografías de plantas en las que se puede observar zonas claras con mayor cantidad de agua.

Esta técnica también se usa para estimar el grado de humedad en maderas [8], tanto de uso industrial (fig.10) como en el árbol (mediante sistemas portátiles) antes de su tala.

En la figura 12a, se muestra la imagen real y la neutrografía de una linterna de inmersión acuática. $\mathrm{Al}$ interior, se observa la pila eléctrica que contiene una pasta electrolítica y sellos de jebe indicada con flechas azules. En la figura 12b, se observa la imagen real y las neutrografias de una bala en posición vertical y horizontal, donde se aprecia la forma de acomodación de la pólvora y el espacio vacío de acuerdo a la posición (flechas rojas).

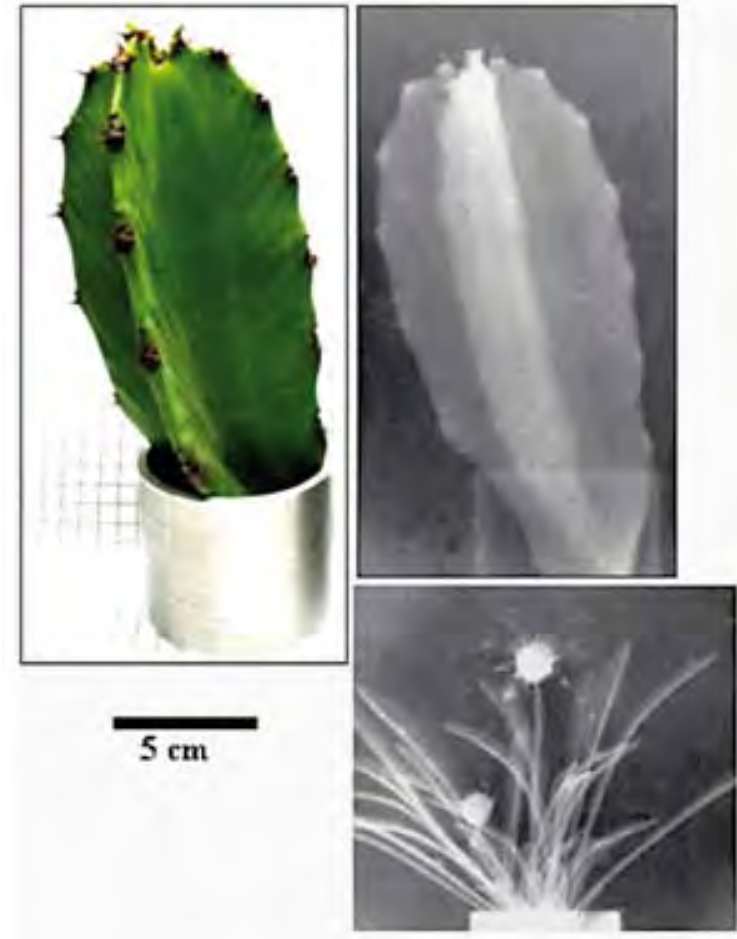

Figura 9: Imagen de luz visible de un cacto con su respectiva neutrografía y planta con un botón y una flor.

a)

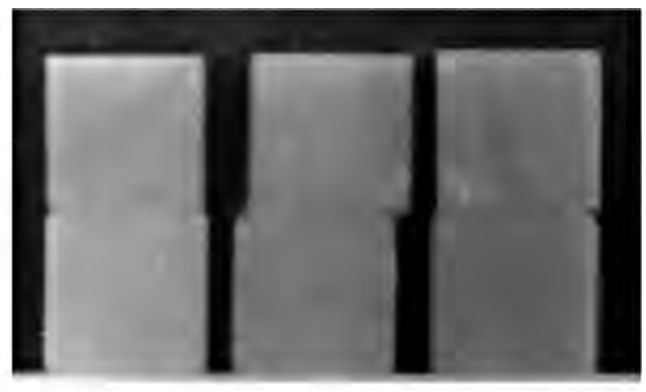

b)
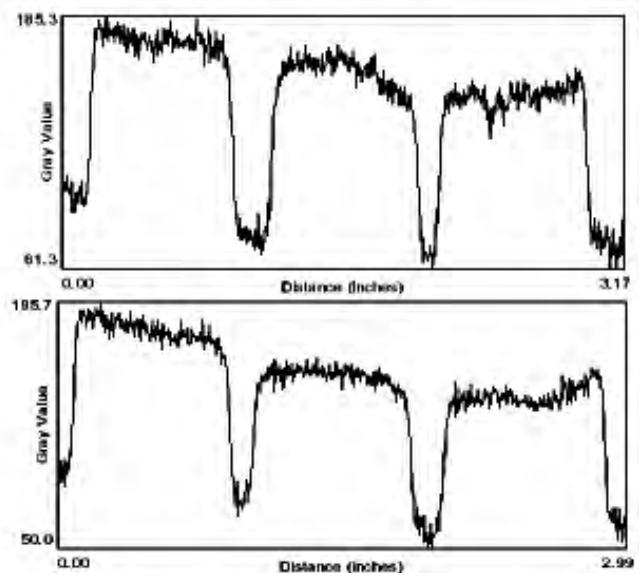

Figura 10: a) Neutrografias de una serie de maderas (mohena, ishpingo y catahua de la amazonia peruana) polimerizadas con PVA e irradiadas con rayos gamma. Las de arriba han sido humedecidas con agua y las de abajo están secas, b) espectros de absorción neutrónica de las dos ternas de maderas secas y humedas, respectivamente. 


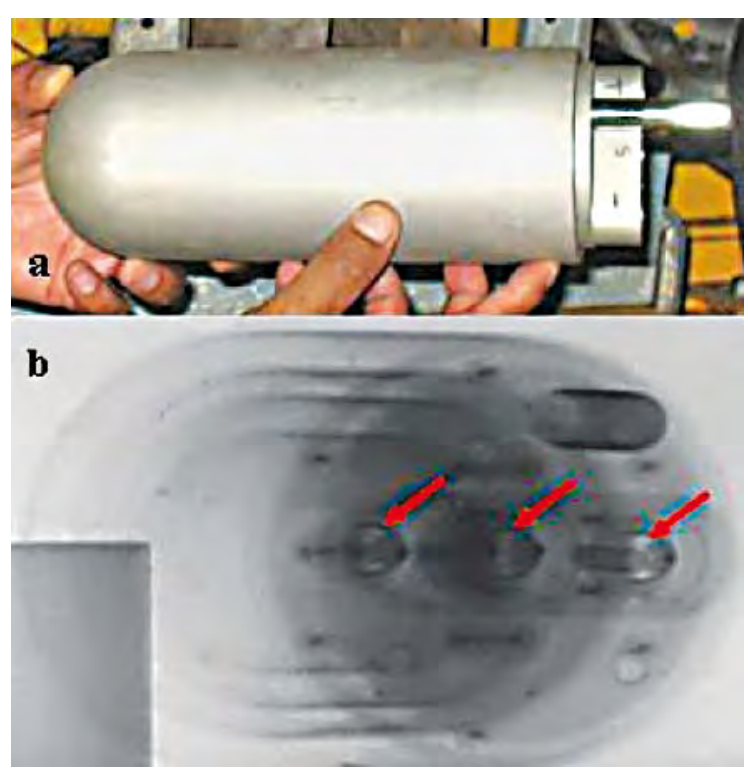

Figura 11: a) Imagen real de una cámara de ionización y b) Neutrografía de dicha cámara en una proyección lateral a $45^{\circ}$, mostrando en alto contraste los sellos aislantes en la base y en los porta electrodos (flechas rojas). Estas imágenes demuestran las ventajas del método, donde las partes metálicas se ven muy transparentes mientras que pequeñas piezas aislantes o plásticas resaltan mucho..

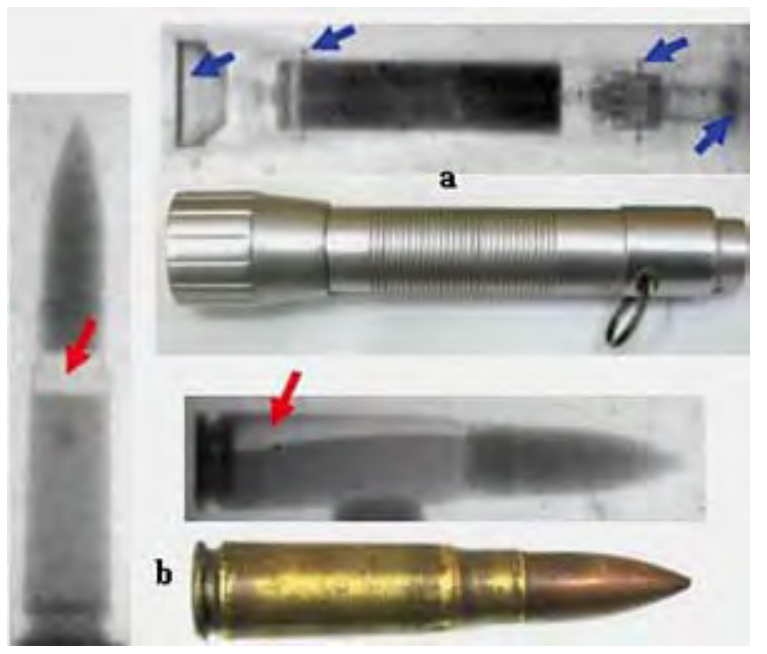

Figura 12: a) Imagen real y neutrografía de una linterna de inmersión acuática, b) Imagen real y neutrografias en posiciones vertical y horizontal de una bala.

En la figura 13, se muestra las neutrografías en tamaño natural y sus correspondientes imágenes reales de piezas arqueológicas metálicas correspondientes al período Intermedio Tardío $(1000$ - 1470 d. C.), de la costa norte del Perú. Como se puede apreciar, las piezas aún no han sido restauradas, motivo por el cual las neutrografías muestran con alto contraste las capas afectadas por la corrosión: a) gancho, b) campanilla.
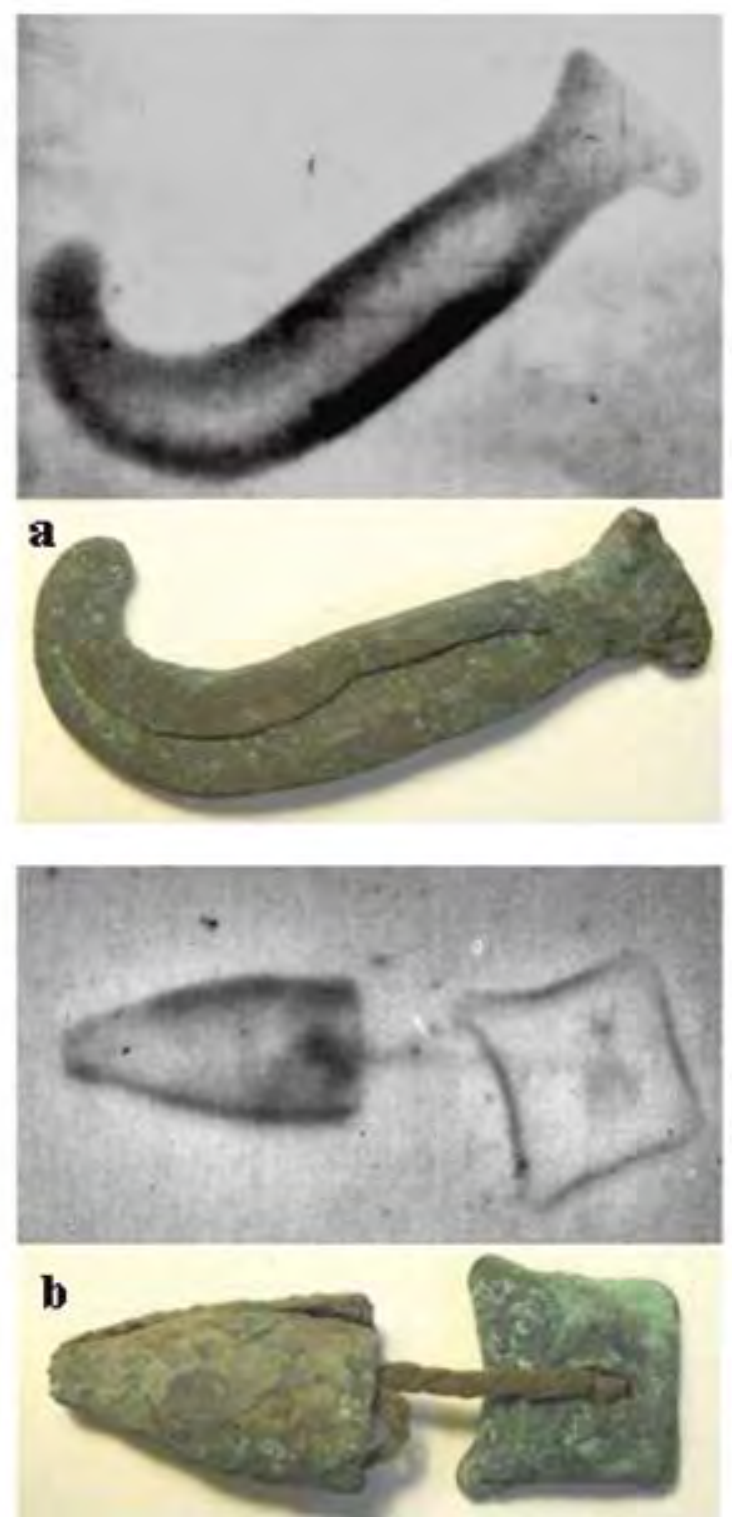

Figura 13: Neutrografias en tamaño natural y sus correspondientes imágenes reales de piezas arqueológicas metálicas: a) gancho, b) campanilla.

\section{CONCLUSIONES}

La neutrografía fue usada para establecer con precisión la ubicación espacial del haz de neutrones de la salida radial número 4 del reactor nuclear RP-10, lo cual nos demuestra su gran potencial para fines de alineamiento de sistemas ópticos neutrográficos, tales como el difractómetro de neutrones, el espectrómetro gamma, etc.

También puede aplicarse a materiales orgánicos. Por ejemplo, puede estudiarse el crecimiento de raíces, la distribución de nutrientes en el suelo y en las plantas, la detección de contaminantes y venenos. Como estos, existen muchos aspectos interesantes 


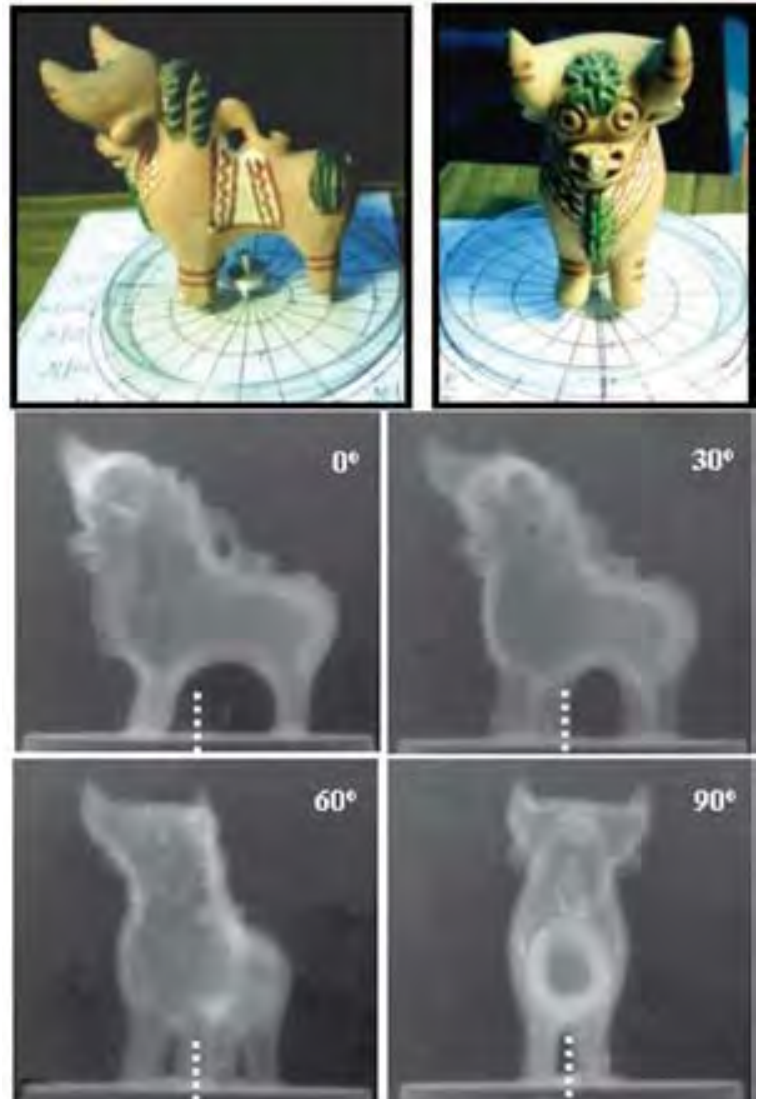

Figura 14: Arriba, imágenes reales de un objeto cerámico. Abajo, neutrografias para estudios de reconstrucción tomográfica en diferentes posiciones de rotación.

para ser investigados en el campo de la biología con la ayuda de los neutrones.

La extremada penetración de los neutrones en los metales hace que las neutrografías tengan ventajas importantes, debido a que las partes metálicas se ven muy transparentes mientras que las pequeñas piezas aislantes o plásticas resaltan mucho.

Otro campo de aplicación importante es el estudio de piezas arqueológicas de nuestra herencia cultural, para ayudar así a una mejor interpretación del pasado y darle mayor valor agregado, mejorando incluso su impacto turístico (fig. 14).

También permiten obtener espectros de absorción neutrónica de muestras de madera sometidas a pruebas de absorción de agua, o localización de pólvora en el interior de las municiones. Las variadas aplicaciones de la neutrografia tendrán un futuro promisorio con el desarrollo de hardware y software para análisis de fases y estudios de procesos dinámicos.
Las pruebas neutrográficas realizadas en las instalaciones del reactor RP-10 del IPEN muestran la capacidad de realizar ensayos de inspección no destructivos usando los neutrones, lo que amplía la capacidad de análisis del reactor RP-10 a un ámbito muy variado. Esta facilidad es ahora extendida a todos los investigadores nacionales y extranjeros (universidades, institutos, industria, etc.), promoviendo tanto la enseñanza como la investigación.

\section{AGRADECIMIENTOS}

Al físico Iván Lovato, por la preparación del software Diffraction usado en el centrado del haz neutrografiado; a la Arqueóloga Luisa Vetter, por proporcionarnos las muestras arqueológicas metálicas.

\section{REFERENCIAS}

[1] http://1.1.1.1/831882256/832131080T10 $1006112835 . t x t . b i n X M y s M 0 d a p p l i c a t i o n /$ pdfXsysM0dhttp://www.oscintl.com/White_ Papers/Troubleshooting_Optical_Alignment_ Problems_Whitepaper_02.19.08.pdf.

[2] IAEA-TECDOC-1604, Neutron Imaging: A Nondestructive Tool for materials testing, IAEA, 2004.

[3] A. M. Shaikh, Development of neutron and $\mathrm{x}$-ray detectors and neutron radiography at Barc Founder's Day Issue 273, 2006.

[4] Wade J. Richards et al., Real-Time Neutron radiography applications for the automotive industry, UCD McClellan Nuclear Radiation Center (Univ. California, Davis), 2003.

[5] Yuri Ravelo R., Caracterización y puesta a punto de la facilidad de neutrografía del reactor Nuclear RP-10, Tesis Univ. Nac. de Ing. Fac. de Ciencias, Lima, 2001.

[6] Marco Munive S., Caracterización en energía de un haz de neutrones térmicos, Tesis Univ. Nac. de Ing. Fac. de Ciencias, Lima, 2000.

[7] Md. Nurul Islam, Development of electronic imaging system for real time neutron radiography at TRIGA MK-II research reactor of AERE, Savar Dhaka and utilization for research \& industrial application IAEA-TECDOC-1604.

[8] Javier Gago et al., Caracterización entre la densidad de maderas amazónicas peruanas mediante la atenuación de la radiación gamma y neutrónica ICT IPEN 2007, 72-78.

[9] R. Pugliesi et al., New radiographic images from old neutron converter screen IAEA-TECDOC-1604.

[10] M. Dinca, The implementation of a charge couplet device (CCD) camera in a neutron imaging system for real time and tomography investigations, IAEATECDOC-1604, 2003, 53.

E-mail: alopez@ipen.gob.pe 